\title{
Are kea prosocial?
}

\section{Megan Heaney $^{1}$ | Amalia P. M. Bastos ${ }^{1}$ (D) | Russell D. Gray ${ }^{1,2,3}$ | Alex H. Taylor ${ }^{1}$}

\author{
${ }^{1}$ School of Psychology, The University of \\ Auckland, Auckland, New Zealand \\ ${ }^{2}$ Max Planck Institute for the Science of \\ Human History, Jena, Germany \\ ${ }^{3}$ School of Philosophy, Australian National \\ University, Canberra, ACT, Australia

\section{Correspondence} \\ Alex Taylor, School of Psychology, The \\ University of Auckland, Private Bag 92019, \\ Auckland 1142, New Zealand. \\ Email: alexander.taylor@auckland.ac.nz

\section{Funding information} \\ This work was supported by a University \\ of Auckland Doctoral Scholarship (MH), a \\ Rutherford Discovery Fellowship (AHT) and \\ a Prime Minister's McDiarmid Emerging \\ Scientist Prize (AHT).
}

\begin{abstract}
Prosocial behaviour is widespread in humans, but evidence for its occurrence in other species is mixed. We presented a parrot species, the kea (Nestor notabilis) with a series of experiments to test whether they exhibit prosocial tendencies. Across the first round of testing, in our first condition, two of the four kea acted prosocially, as they preferred to choose a prosocial token which rewarded both themselves and a partner, rather than a token that rewarded only themselves. Three of the four kea then showed a preference for the prosocial token in a second condition where they alternated taking turns with a partner. However, no kea showed a decrease in the third yoked control condition in which the experimenter replicated the token choice made by the partner in the previous alternating trials. This yoked condition was used to dissociate truly reciprocal behaviour, whereby the actor made choices based on their partner's choices, from a response to the amount of rewards conferred to the partner. Finally, three of the four kea continued to choose the prosocial token in the fourth asocial control condition where no partner was present. However, in round two of testing, one kea changed its token choices to a similar pattern to that expected if kea are prosocial, in that it preferred the prosocial token in the initial condition, showed a trend for the prosocial token when turns were alternated, but chose at chance in the yoked and asocial conditions. This study therefore found no evidence of spontaneous reciprocity in kea but further testing is required before we can conclude that kea are not capable of prosocial behaviour at all.
\end{abstract}

\section{KEYWORDS}

giving assistance test, kea, parrot, prosociality, token exchange

\section{1 | INTRODUCTION}

Prosocial behaviour, whereby an individual confers benefits to another at no cost to themselves, is ubiquitous in humans and is likely to have played a role in the evolution of human cooperative behaviour (Amici et al., 2014; Horner, Carter, Suchak, \& Waal, 2011; Melis et al., 2011). Children develop spontaneous prosociality before they begin to take partner's actions into account and reciprocate accordingly (contingent reciprocity) (House et al., 2013; Sebastián-Enesco \& Warneken, 2015; Warneken \& Tomasello, 2013). Animals also demonstrate behaviours indicative of prosocial tendencies in naturalistic settings, such as mutual grooming, sharing food, assistance in conflict and cooperative hunting (Koyama, Caws, \& Aureli, 2006; Schino, 2007; de Waal, 1997).

Prosociality in animals has typically been examined using two different paradigms, the Giving Assistance Test (GAT) and the Prosocial Choice Test (PCT). In the GAT, subjects must decide between giving instrumental help to another and doing nothing. GAT studies have demonstrated that chimpanzees were willing to provide assistance to a conspecific by giving them a tool they required to access a reward (Yamamoto, Humle, \& Tanaka, 2009, 2012), unlocking a door that prevented a partner from accessing a food reward (Warneken, Hare, Melis, Hanus, \& Tomasello, 2007, but see Tennie, Jensen, \& Call, 2016), releasing food so it could be reached by a conspecific 
(Melis et al., 2011) and pulling a handle that helped the conspecific access the reward (Greenberg, Hamann, Warneken, \& Tomasello, 2010).

In PCT tasks, subjects must choose between a selfish option $(1 / 0)$ which only yields a reward for the subject, and a prosocial option $(1 / 1)$ that yields a reward for both subject and partner. For the most, PCT tasks have examined prosociality in animals using tokens or platforms (Amici et al., 2014; Marshall-Pescini, Dale, QuervelChaumette, \& Range, 2016). Selection of the selfish token or platform only confers a reward to the subject, whereas selection of the prosocial token or platform confers a reward to both subject and their partner.

One of the earliest PCT studies showing evidence for prosociality in non-human animals was conducted in capuchins (Cebus apella) (de Waal, Leimgruber, \& Greenberg, 2008), but was subsequently criticized for its lack of essential controls (House, 2014; Amici et al., 2014; Jensen, Hare, Call, \& Tomasello, 2006). The original experimental designs used in prosociality studies (Colman, Liebold, \& Boren, 1969; Nissen \& Crawford, 1936) remain controversial due to their lack of partner-absent controls, as results cannot dissociate between prosocial behaviour and a learnt preference for the prosocial choice irrespective of context (Amici et al., 2014; Marshall-Pescini et al., 2016). However, since then several studies have run appropriate controls (Silk et al., 2005; Jensen et al., 2006; Vonk et al., 2008; Cronin, Schroeder, \& Snowdon, 2010; Massen, Van den Berg, Spruijt, \& Sterck, 2010; Stevens, 2010; Massen, Lambert, Schiestl, \& Bugnyar, 2015; Suchak \& de Waal, 2012; Takimoto, Kuroshima, \& Fujita, 2010; Drayton \& Santos, 2014; Quervel-Chaumette, Dale, Marshall-Pescini, \& Range, 2015; Horn, Bugnyar, \& Massen,2019).

One prominent example is a study by Suchak and de Waal (2012), who investigated prosociality in capuchin monkeys, seeking to investigate: (a) if prosociality increased when subjects took turns, and (b) if this increase was based on contingent, or non-contingent reciprocity. Additionally, subjects were tested in two conditions, an alternating turns condition and a yoked control condition, which were designed to assess whether an increase in prosocial preference in the alternating turns condition was due to prosocial behaviour or simply due to the rewards conferred by the prosocial token. These conditions directly tested not only whether capuchins act prosocially, but also whether they are able to take their partner's choices into account in a reciprocal task, and so demonstrate contingent reciprocity. The alternating condition tested whether a subject's choice was affected by their partner's choices. If so, the yoked condition served to dissociate a response to the partner's behaviours from a response to the rewards obtained, irrespective of the partner's behaviour. In the yoked condition, the partner was present but did not take part in the trials. Instead, the experimenter made the same choices as the actor had previously made in their alternating condition trials.

Suchak and de Waal (2012) found that the capuchins acted prosocially, with prosocial tendencies increasing significantly when they alternated taking turns with their partner. However, token choice was not contingent upon the choice that their partner had made in the previous trial, suggesting that subject's choices were not based on contingent reciprocity but were instead due to subjects being prosocial irrespective of the actions of their partner.

Whilst most prosociality studies have focused on mammals, particularly primates, it is also important to study prosociality in distantly related species as it allows us to examine whether prosociality can evolve convergently, and so discover what selective pressures might be responsible for its evolution (Smith, Shields, \& Washburn, 2003). Given that prosocial tendencies in primates are often context-dependent and weaker in comparison with humans (Burkart et al., 2014; Skoyles, 2011), studying other taxa could potentially shed light on its evolution. Birds, particularly parrots and corvids, are an excellent choice for such comparisons, given how distantly related they are to humans (Benton \& Donoghue, 2007). They both live in social groups, have extended periods of parental dependence and have large forebrains relative to their size (Bond \& Diamond, 2003; Emery, Seed, Bayern, \& Clayton, 2007). So far, only a few studies explored prosociality in birds, with little evidence of other-regarding preferences in the latter (Schwab, Swoboda, Kotrschal, \& Bugnyar, 2012; Péron, John, Sapowicz, Bovet, \& Pepperberg, 2013; Di Lascio, Nyffeler, Bshary, \& Bugnyar, 2013; Horn et al.,2019; Wascher, Feider, Bugnyar, \& Dufour,2019).

To date, four studies in corvids have shown no evidence for prosocial preferences (Di Lascio et al., 2013; Massen et al., 2015; Lambert, Massen, Seed, Bugnyar, \& Slocombe, 2017; Wascher et al.,2019 ). Jackdaws (Coloeus monedula) made more prosocial choices when their partner was positioned near the prosocial option before they made their choice (Schwab et al., 2012), but, as the authors suggest, this result could be caused by stimulus enhancement rather than prosocial tendencies. So far, only two corvid studies show spontaneous prosocial behaviour in azure-winged magpies, Cyanopica cyanus, (Horn, Scheer, Bugnyar, \& Massen, 2016) and pinyon jays, Gymnorhinus cyanocephalus (Duque, Leichner, Ahmann, \& Stevens, 2018).

In psittacids, a single study in African grey parrots (Psittacus erithacus) provided some evidence for reciprocity, rather than prosociality. This was observed only in a single individual, whose reciprocity depended on the order in which they interacted with their partner (Péron et al., 2013).

Kea (Nestor notabilis) have been successful at solving problems in the domains of both technical and social cognition (Auersperg, Gajdon, \& Huber, 2010; Auersperg, Huber, \& Gajdon, 2011; Diamond \& Bond, 1999; Huber \& Gajdon, 2006; Tebbich, Taborsky, \& Winkler, 1996), including cooperative tasks (Schwing, Jocteur, Wein, Noë, \& Massen, 2016, this issue; Heaney, Gray, \& Taylor, 2017a; Heaney, Gray, \& Taylor, 2017b), making them a suitable candidate for prosocialitystudies. Here, we examined prosociality in kea in four different conditions using the same paradigm as Suchak and de Waal (2012). Condition 1 examined whether kea exhibited a spontaneous prosocial choice, and so preferred to choose a prosocial token, which rewarded both the subject and a partner, over a selfish one, which rewarded only the subject. In Condition 2, kea took turns choosing a token with a partner. This condition examined whether kea might make prosocial decisions contingent upon a conspecific partner's 
actions. Condition 3 was a yoked control condition in which the focal kea was the only one to select a token and the experimenter replicated the choice of the partner from previous trials in condition 2. This tested whether kea was responding to the choices their partner made in condition 2 or to the rewards they were receiving. If they were responding to their partner's choices, prosocial choices would be expected to decrease in condition 3 as the experimenter, rather than the partner, was choosing, despite the rewards being received within each condition being identical. Finally, subjects were tested in a partner-absent condition. This assessed whether they had simply developed a strong preference for a particular token that was independent of whether a partner was actually present.

\section{2 | METHODS}

\section{1 | Subjects}

We tested four male kea, aged between one and three years. Neo, Zak and Taz were captive bred at Willowbank Wildlife Reserve in Christchurch, and Bruce was born in the wild and came to Willowbank as a fledgling. Subjects share a large outdoor aviary with nine other kea. Subjects were tested in pairs (see Table 1) within their aviary and were free to come and go from the testing apparatuses at any time. Pairs were selected on the basis of birds that had previous experience with the apparatus and had demonstrated a tolerance for being inside the apparatus with their partner on the other side. Only four birds were suitable for testing, as they would voluntarily enter the apparatus provided and could be selected for individual training and testing within the enclosure. If other subjects approached the testing area, they were led away with food. Food and water were available ad libitum within the aviary. This study was conducted under approval from the University of Auckland animal ethics committee (reference no. 001416).

\section{2 | Apparatus}

Subjects were trained and tested in a wooden apparatus $(150 \mathrm{~cm} \times 50 \mathrm{~cm} \times 100 \mathrm{~cm}$ ) (Figure 1). The apparatus had a wooden frame covered in chicken wire enabling subjects to see each other but not interfere with their partner's behaviour. The top of the apparatus was not covered so that subjects could exit the apparatus at any point and could enter at two separate entry points at opposite ends. As in previous experiments, both subjects had to wait for a wooden partition to be removed before they could enter the apparatus at the start of each trial (Heaney et al., 2017a, 2017b). Pieces

TABLE 1 Subject dyads tested in both rounds of the experiment. Both subjects within each pair acted as both the actor and the receiver in all conditions of the study. All subjects were adult males

\begin{tabular}{ll} 
Round $\mathbf{1}$ & Round $\mathbf{2}$ \\
\hline Taz + Neo (siblings) & Neo + Zak (siblings) \\
Bruce + Zak (unrelated) & Bruce + Taz (unrelated) \\
\hline
\end{tabular}

of wooden dowel $(5 \mathrm{~cm} \times 3 \mathrm{~cm})$ dyed with food colouring were used as tokens for subjects to exchange, with the exception of Bruce's tokens which were not as wide $(5 \mathrm{~cm} \times 1.5 \mathrm{~cm})$ due to him missing his upper mandible. During testing, tokens were placed in plastic containers $(20 \mathrm{~cm} \times 17 \mathrm{~cm} \times 10 \mathrm{~cm})$ which were attached at the rear of the interior of the apparatus.

\section{3 | Preliminary tasks and familiarisation}

\subsection{1 | Token exchange training}

All subjects were naïve at token exchange paradigms. In stage 1, kea learned to exchange tokens. Tokens for this stage were naturally coloured pieces of dowel measuring $5 \times 3 \mathrm{~cm}$. Kea were required to enter the apparatus, where a token was placed in front of the experimenter. Kea had to learn to put the token in a small platform just beside the token which was accessible to both the subject and the experimenter. Following this, the experimenter retrieved the token and immediately rewarded the kea. Throughout both training and testing, the first token to be touched to the platform was considered the subject's final choice. Training continued until kea placed the token in the platform 10 times in a row.

\subsection{2 | Token preference test}

Following token exchange training, subjects were given a token preference test similar to that designed by (de Waal and colleagues 2008) to make sure that they did not have a significant preference for a certain colour. Subjects were presented with two differently coloured tokens side by side for 10 trials, and subjects had to choose

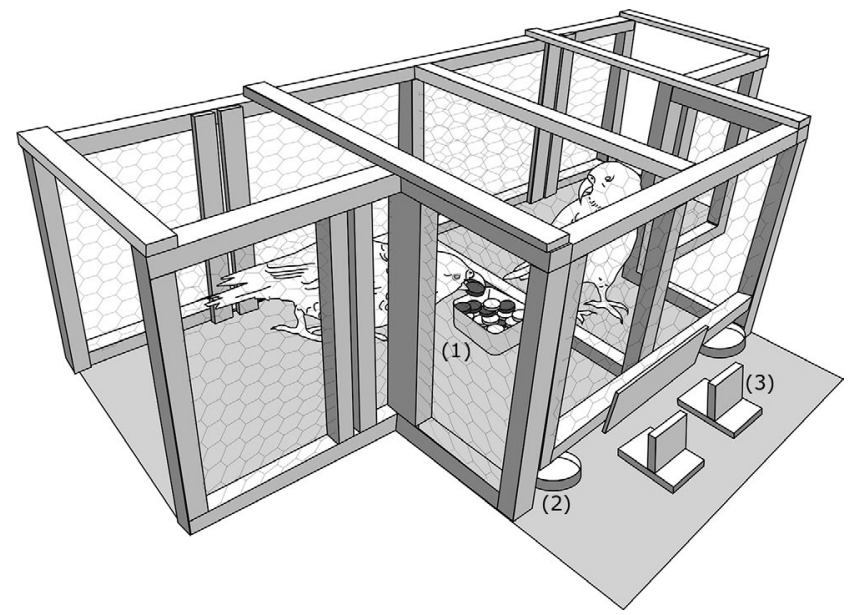

FIGURE 1 Experimental setup and apparatus. One kea selects either a prosocial or selfish token from the token box (1) and places it in the small dish (2) on their side. The experimenter takes the token and places it in front of both kea, then retrieves the corresponding reward from behind the barrier on the platform (3) and places it in full view of the kea. A reward for the subject, or rewards for both the subject and their partner are then delivered to the kea by sliding the platforms underneath the gap in the apparatus. Drawing by Vivian Ward 
one token to trade with the experimenter for a reward, gaining one piece of Hills Science Diet for each token. If any subjects had a preference for one token in 8 or more of the 10 trials, both tokens would be replaced with new colours and neither colour would be used during testing. The average initial preference for any token colour was $52.5 \pm 13.89 \%$, and no token needed to be replaced. Subjects were tested with six different colours (purple/green, blue/pink and orange/black).

\subsection{3 | Training on token values}

Stage 2 of training was designed to familiarise kea with different token values. Three different token colours were trained for each round. These were an unrewarding token $(0 / 0)$, in which neither the subject or partner received a reward if it was selected, a prosocial token (1/1), whereby both subject and partner received a reward and a selfish token (1/0), in which only the subject received a reward. In any one trial only two tokens were used. The unrewarding token was used in every trial and was paired with either the selfish token or the prosocial token. Kea received trials (unrewarding with selfish or unrewarding with prosocial) in randomized order and had to learn to discriminate between the two tokens. Rewards were placed out of subjects' view behind a screen. There were two identical platforms, one in front of the subject and one in front of the partner. Once the subject placed a token in the platform, the experimenter either retrieved a reward from behind either both screens if the prosocial token was returned, or only the subject's screen if the selfish token was returned, or no reward if the unrewarding token was selected. Rewards were held up so that they were now visible to both kea, placed on one or both platforms in front of the screen and then slid towards either both subject and partner or just the subject. After ten consecutive successful trials of choosing the selfish token over the unrewarding token and the prosocial token over unrewarding token, kea moved onto stage 3.

Stage 3 of training was designed to teach the kea they were now required to select tokens from a plastic container at the back of the apparatus and place it in the platform in front of the experimenter. Kea were presented with token combinations (unrewarding and selfish, or unrewarding and prosocial) jumbled in a container with 6 tokens, 3 of each option, in pseudorandomised order. Kea had to select either the selfish token over the unrewarding token or the prosocial token over the unrewarding token in 10 trials in a row. After ten successful trials, subjects continued onto stage 4 of training.

Stage 4 was designed to further test that the kea had learnt the value of each token. Previous PCT experiments using token exchange paradigms have used subjects that had extensive previous experience with token exchange paradigms. Subjects were given pseudorandomised trials where they either had to take a selfish token (forced choice selfish trials, 10 given in total), choose between an unrewarding and selfish token ( 5 trials) or choose between an unrewarding and prosocial token (5 trials). Ten successful exchanges in trials that included the unrewarding tokens were required to proceed to testing. For stage 4 , subjects received between two and three sessions of 30 trials per day and took between one and three days to complete training.

\subsection{General procedure}

\subsection{1 | Description of conditions}

The experiment consisted of four different conditions which were identical to that used by Suchak and de Waal (2012):

\section{Condition 1-Spontaneous condition}

This condition tested whether kea would demonstrate a spontaneous prosocial choice. In this condition, only one of the keas was able to choose the tokens.

\section{Condition 2-Alternating condition}

This condition assessed prosocial preference when kea took alternating turns at selecting a token with a partner. This condition tested whether prosociality increased in situations in which reciprocity is possible.

\section{Condition 3-Yoked condition}

In this condition, the subject selected a token. However, instead of the partner selecting a token on the next trial, the experimenter chose the token that the partner had chosen in the corresponding alternating session. The partner was still present in the next door apparatus but did not make a choice. This condition was designed to test whether any increase in prosocial token preference in the alternating condition was due to kea responding to what their partner actually chose in the alternating condition or simply the rewards conferred by their partner's choices. Prosocial choice between the alternating and yoked conditions would be expected to decrease if kea were responding to their partner's choices and behaviour rather than just the outcomes of their partner's token choice, as subject' choices could not affect their partner's behaviour in the yoked condition.

\section{Condition 4-Partner-Absent condition}

This condition was designed to assess whether an observed prosocial preference in kea was due to them possessing other-regarding preferences, or whether it was based on them having developed a strong preference for the prosocial token. This condition was exactly the same as the spontaneous condition, except that the partnering kea was absent.

\subsection{2 | Testing}

There were two rounds of testing. In round one, subjects were paired with the same partner and completed all four conditions in the same order (spontaneous, alternating, yoked and partnerabsent conditions). In the second round, we changed the existing dyads, so that the more dominant bird remained on the same apparatus whilst their partner moved to the second apparatus. Again, conditions were presented in the same order. This order 
was required due to our study design. For example, the yoked condition could not be run until the alternating condition has been run, as it required this data for its execution. Token colours were also different between rounds. Training and testing were identical for both rounds, with one kea acting as the subject from stage 2 of training until the end of condition 1, then the two subjects roles being reversed. At this point, the other kea underwent all the training stages and completed condition 1 . Subjects were then paired up again and given round two of testing. Pairs were therefore kept the same throughout the remaining conditions within each round, with subjects tested in turns. This gave subjects more opportunity to understand the contingencies of each condition. In each condition, subjects had to choose one token from a container filled with 10 tokens, 5 prosocial tokens (1/1) and 5 selfish tokens $(1 / 0)$. After selecting a token, subjects were required to place it in a platform in front of the experimenter. The experimenter then retrieved the token and placed it in front of both kea. Rewards were retrieved from behind the screen attached to the platform, held up for both kea to observe, placed on the platform and delivered to subjects. The actor always received their reward first. Tokens were replaced and jumbled after every trial. Rewards were one piece of Hills Science Diet for each kea. Kea received 3 sessions of 20 trials per condition, and subjects received between one and two sessions a day.

During round 2 , session 2 of the alternating turns condition, two subjects (Neo and Zak) began to enter the apparatus, take a token and then leave the apparatus with it and disperse it throughout the aviary, which eventually became disruptive. To continue testing, we attached one end of a piece of string $(40 \mathrm{~cm})$ to each token and to the top of the apparatus. The string was long enough so that it did not get tangled when subjects were selecting a token, and the experimenter was still able to jumble the tokens after each trial. We tested both subjects like this for one session (Session 2, round 2), after which we resumed testing without the strings attached. This did not affect token preference, as Neo exclusively chose the prosocial token in all three sessions and Zak chose the prosocial token in 5 out of 10 trials of each session.

\subsection{3 | Analyses}

All trials were coded in situ and filmed. Results were analysed the individual level, so we could see if any kea had performed as expected if kea have the capacity to be prosocial. Statistical analyses were carried out in R 3.5.3 (R Core Team, 2014).

\section{3 | RESULTS}

In round 1 of condition 1, prosocial token preference ranged from $11.67 \%$ to $78.33 \%$ across subjects (mean \pm SEM: $52.92 \pm 16.03 \%$ ), whilst in round 2 this ranged from $40 \%$ to $85 \%$ (mean \pm SEM: $59.58 \pm 11.17 \%)$. Prosocial token preference per round of testing is shown in Figure 2.

Individual performance per condition in each round of testing is summarised in Table 2. Bruce and Taz both chose the prosocial token significantly above chance in all conditions of round 1 (Figure 3). Neo had a significant preference for the selfish token in the spontaneous condition, then switched his preference to the prosocial token for the alternating and yoked conditions. He then chose at chance in the absent partner control condition. Zak only selected the prosocial token significantly above chance in the absent partner condition, whilst in all other conditions, his preference was not significantly above chance.

Performance in round 2 (Figure 4) differed from that in the first round for all subjects. Taz showed a pattern of behaviour consistent with prosociality, significantly preferring the prosocial token in spontaneous condition, trending towards significance in the alternating condition ( $p=.073$ ), but not in the two control conditions. In contrast, Neo showed a significant preference for the prosocial token in every condition for the second round of testing, whilst Zak chose the prosocial token significantly above chance only in the two control conditions. Bruce selected the prosocial token at chance for the first three conditions, and preferred the selfish token significantly above chance in the absent partner control condition.

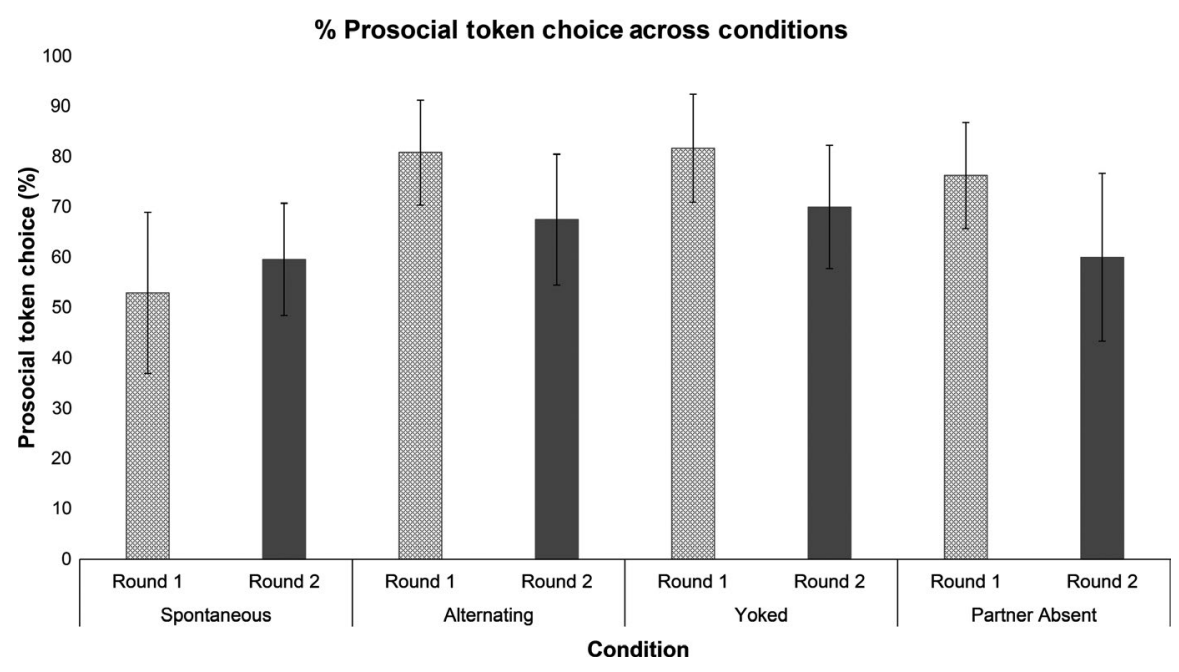

FIGURE 2 Comparison of prosocial choices across conditions. The mean \pm standard error of the mean per cent of prosocial choices are illustrated. Conditions are shown in the order in which they were presented to subjects in both rounds 
TAB LE 2 Subjects' performance in each round of testing for the four conditions: spontaneous, alternating, yoked and absent partner

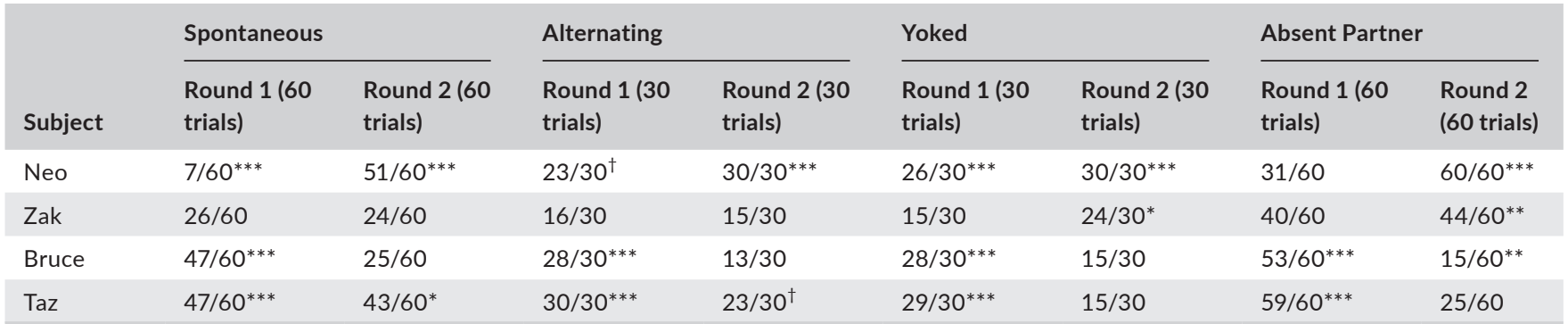

Note: All $p$-values were calculated using a two-tailed binomial test with relative preference of 0.5 and corrected for multiple comparisons using Holm-Bonferroni corrections. Green text indicates a significant preference for the prosocial token, whilst red text indicates a significant preference for the alternative choice. Black text indicates no significant difference from chance.

${ }^{*} p<.05$.

$*^{* *} p<.01$.

${ }^{* * *} p<.001$.

${ }^{\dagger} p<.08$, trending towards significance at $p<.05$ after Holm-Bonferroni corrections, $p<.05$ without alpha corrections.

FIGURE 3 Percentage prosocial token choice for round 1 , across the four conditions for each subject. Conditions are shown in the order in which they were presented to subjects at test

FIGURE 4 Percentage prosocial token choice for round 2 , across the four conditions for each subject. Conditions are shown in the order in which they were presented to subjects at test
$\%$ Prosocial token choice across conditions - Taz per individual in round 1

$\because \mathrm{NeO}$

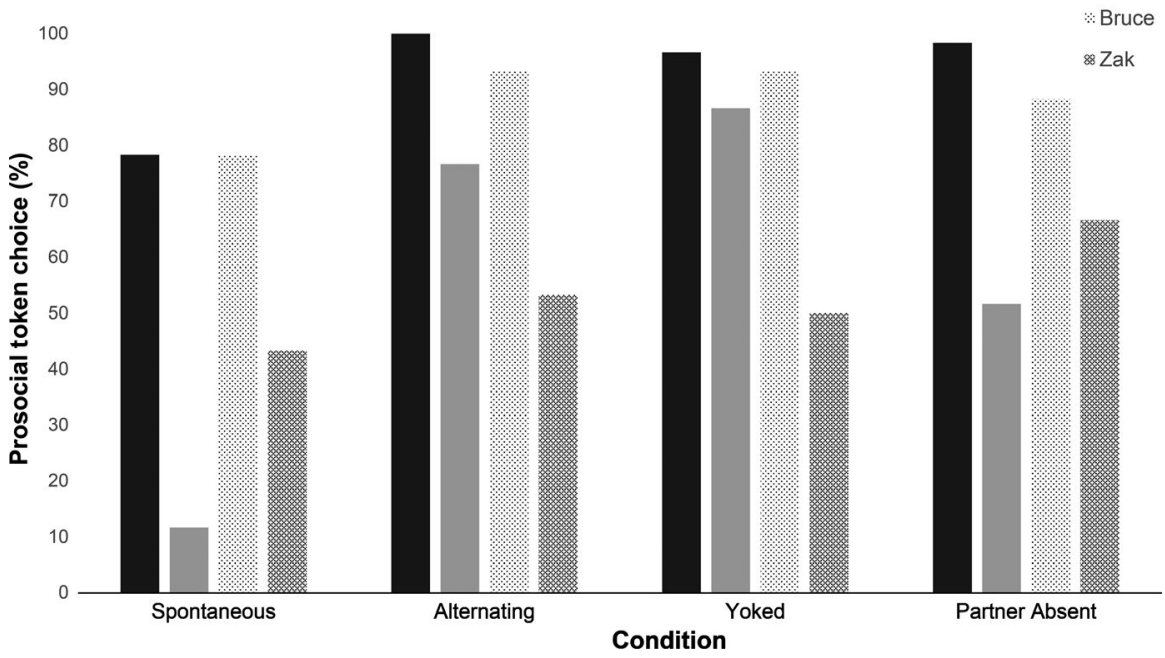

$\%$ Prosocial token choice across conditions per individual in round 2

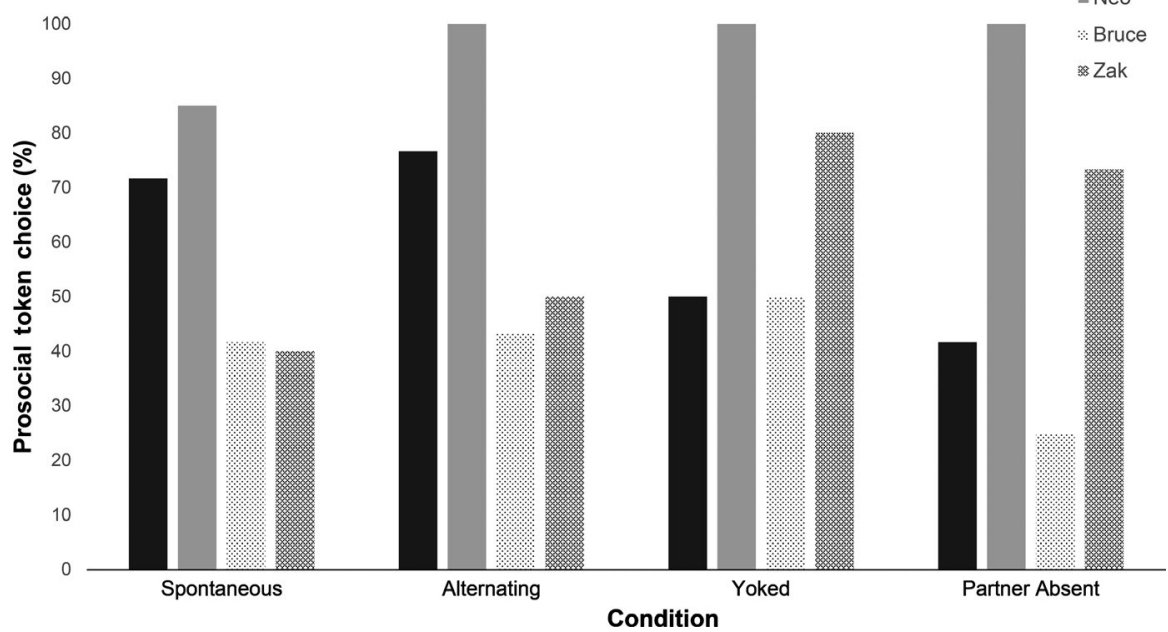


Whilst Suchak and de Waal (2012) found no contingency between the choices that a subject made and the choice that had previously been made by their partner in the alternating condition, we found evidence of a significant contingency between actor's choices and that made by their partner (Pearson's Chi, $\chi^{2}=5.771, p=.016$ ), suggesting that actors changed their behaviour in response to the choices made by their partners. However, these results were in the opposite direction from that predicted by contingent reciprocity, with actor's selfish token choices more likely to follow after their partner's prosocial token selection (Pearsons $r=-.17, n=4, p=.010$ ). Kea were therefore more likely to act selfishly if their actor had previously made a prosocial choice in the previous trial.

\section{4 | DISCUSSION}

Taken together, our results do not suggest that kea are spontaneously prosocial, but may behave prosocially after learning the contingencies of a PCT task. Although two kea showed a preference for the prosocial token in the spontaneous condition in the first round of testing, neither flexibly switched their preferences in the yoked or absent partner conditions in that same round. In fact, three out of four kea chose the prosocial token significantly above chance in the absent partner control condition in the first round of testing, and two chose it in the second round of testing. This may be because the prosocial token was associated with the visible delivery of twice as many food rewards compared with the selfish token, even though the subject always received the same amount of food. This association could have made the prosocial token more rewarding than the selfish token, irrespective of the subjects' understanding of the task. This is especially likely given that the selfish token did not confer any benefits to the actor, relative to the prosocial token. Therefore, there was no incentive to select the selfish token over the prosocial one, and subjects may have simply continued to select the prosocial token throughout testing rounds.

A single kea, Taz, switched his preferences between the first and second rounds of testing, selecting the prosocial token above chance in the spontaneous condition, selecting it nearly above chance in the alternating condition of the second round of testing, but at chance in the yoked and absent partner control conditions. This pattern of results is close to what would be expected if Taz was acting prosocially, though there is some ambiguity given his performance in the alternating condition. Alternatively, given this pattern was seen in only one kea, it could also have been generated by chance. Previous studies of prosocial behaviour have looked only at the final round of testing (Burkart et al., 2014; Horn et al., 2016; de Waal et al., 2008) or compared between multiple rounds of testing (Péron et al., 2013), as in some cases subjects took several rounds to learn the contingencies of the task, and only switched their behaviour after gaining sufficient experience. Similarly, kea may have required several rounds of testing to understand the contingencies of a PCT task and begin making prosocial choices. This may be especially the case with a population that was originally naïve to token exchange paradigms, which was not the case for most primate species tested. Thus, it remains possible that more kea might have acted prosocially if we had run a third round of testing and so further testing in kea is therefore needed before we can conclude that kea show no evidence of prosocial tendencies.

A potential issue with our study was that it only investigated two actor-partner combinations, one for each round of testing. However, studies on primates have shown (Marshall-Pescini et al., 2016; Massen, Luyten, Spruijt, \& Sterck, 2011) that dominance hierarchies may play a role in the emergence of prosocial behaviour. An increased sample size and a variety of actor-partner dyads may have increased the likelihood of prosocial interactions in this task.

Given the intriguing behaviour of Taz, further work is clearly required to before we can conclude that kea are not capable of prosocial behaviour. Multiple rounds of testing would allow subjects to gain a better understanding of the contingencies of the task and establish whether or not Taz's performance was driven by prosocial tendencies or chance. Comparison of performance with paradigms which do not involve food would also be useful (Jensen et al., 2006; Quervel-Chaumette et al., 2015; Warneken et al., 2007), as would testing with more ecologically-relevant scenarios (Cronin, 2012; Marshall-Pescini et al., 2016).

\section{ACKNOWLEDGEMENTS}

We thank the staff at Willowbank Wild Reserve, in particular, Nick Ackroyd and the staff at the Natives section, for their invaluable help and cooperation with our research, and Vivian Ward for creating our figures. We also thank two anonymous reviewers for their comments on the manuscript.

\section{CONFLICT OF INTERESTS}

The authors declare no competing interests.

\section{AUTHOR CONTRIBUTIONS}

$\mathrm{MH}, \mathrm{RDG}$ and $\mathrm{AHT}$ designed the experiments; $\mathrm{MH}$ collected data; APMB analysed the data; MH, APMB, RDG and AHT wrote the paper. All authors gave approval for publication.

\section{ETHICAL APPROVAL}

This research was conducted under ethics approval from The University of Auckland Ethics Committee (reference number 001416).

\section{ORCID}

Amalia P. M. Bastos (iD https://orcid.org/0000-0003-3037-687X

\section{REFERENCES}

Amici, F., Aureli, F., Mundry, R., Amaro, A., Barroso, A., Ferretti, J., \& Call, J. (2014). Calculated reciprocity? A comparative test with six primate species. Primates, 55, 447-457. 
Auersperg, A. M. I., Gajdon, G. K., \& Huber, L. (2010). Kea, Nestor notabilis, produce dynamic relationships between objects in a secondorder tool use task. Animal Behaviour, 80, 783-789.

Auersperg, A. M. I., Huber, L., \& Gajdon, G. K. (2011). Navigating a tool end in a specific direction: Stick-tool use in kea (Nestor notabilis). Biology Letters, 7, 825-828.

Benton, M. J., \& Donoghue, P. C. J. (2007). Paleontological evidence to date the tree of life. Molecular Biology and Evolution, 24, 26-53.

Bond, A., \& Diamond, J. (2003). A comparative analysis of social play in birds. Behaviour, 140, 1091-1115.

Burkart, J. M., Allon, O., Amici, F., Fichtel, C., Finkenwirth, C., Heschl, A., ... van Schaik, C. P. (2014). The evolutionary origin of human hyper-cooperation. Nature Communications, 5. https://doi.org/10.1038/ncomm s5747

Colman, A. D., Liebold, K. E., \& Boren, J. J. (1969). A method for studying altruism in monkeys. The Psychological Record, 19, 401-405.

Cronin, K. A. (2012). Prosocial behaviour in animals: The influence of social relationships, communication and rewards. Animal Behaviour, 84, 1085-1093.

Cronin, K. A., Schroeder, K. K. E., \& Snowdon, C. T. (2010). Prosocial behaviour emerges independent of reciprocity in cottontop tamarins. Proceedings of the Royal Society B: Biological Sciences, 277(1701), 3845-3851. https://doi.org/10.1098/rspb.2010.0879

de Waal, F. B. M. (1997). The Chimpanzee's service economy: Food for grooming. Evolution and Human Behavior, 18, 375-386.

de Waal, F. B. M., Leimgruber, K., \& Greenberg, A. R. (2008). Giving is self-rewarding for monkeys. Proceedings of the National Academy of Sciences of the United States of America, 105, 13685-13689.

Di Lascio, F., Nyffeler, F., Bshary, R., \& Bugnyar, T. (2013). Ravens (Corvus corax) are indifferent to the gains of conspecific recipients or human partners in experimental tasks. Animal Cognition, 16, 35-43.

Diamond, J., \& Bond, A. (1999). Kea, bird of paradox: The evolution and behavior of a New Zealand parrot. Berkely, CA: University of California Press.

Drayton, L. A., \& Santos, L. R. (2014). Insights into intraspecies variation in primate prosocial behavior: Capuchins (Cebus apella) fail to show prosociality on a touchscreen task. Behavioral Sciences (Basel, Switzerland), 4, 87-101.

Duque, J. F., Leichner, W., Ahmann, H., \& Stevens, J. R. (2018). Mesotocin influences pinyon jay prosociality. Biology Letters, 14(4), https://doi. org/10.1098/rsbl.2018.0105

Emery, N. J., Seed, A. M., von Bayern, A. M. P., \& Clayton, N. S. (2007). Cognitive adaptations of social bonding in birds. Philosophical Transactions of the Royal Society B: Biological Sciences, 362, 489-505.

Greenberg, J. R., Hamann, K., Warneken, F., \& Tomasello, M. (2010). Chimpanzee helping in collaborative and noncollaborative contexts. Animal Behaviour, 80, 873-880.

Heaney, M., Gray, R. D., \& Taylor, A. H. (2017a). Kea show no evidence of inequity aversion. Royal Society Open Science, 4(3). https://doi. org/10.1098/rsos.160461

Heaney, M., Gray, R. D., \& Taylor, A. H. (2017b). Keas perform similarly to chimpanzees and elephants when solving collaborative tasks. PLoS One, 12, e0169799.

Horn, L., Bugnyar, T., \& Massen, J. J. (2019). No proof for prosocial preferences in carrion/hooded crows' when tested in a token exchange paradigm. Ethology.

Horn, L., Scheer, C., Bugnyar, T., \& Massen, J. J. M. (2016). Proactive prosociality in a cooperatively breeding corvid, the azure-winged magpie (Cyanopica cyana). Biology Letters, 12(10). https://doi.org/10.1098/ rsbl.2016.0649

Horner, V., Carter, J. D., Suchak, M., \& de Waal, F. B. M. (2011). Spontaneous prosocial choice by chimpanzees. Proceedings of the National Academy of Sciences of the United States of America, 108, 13847-13851.
House, B. (2014). Task design influences prosociality in captive chimpanzees (Pan troglodytes). PLoS One, 9, e103422.

House, B. R., Silk, J. B., Henrich, J., Barrett, H. C., Scelza, B. A., Boyette, A. H., ... Laurence, S. (2013). Ontogeny of prosocial behavior across diverse societies. Proceedings of the National Academy of Sciences of the United States of America, 110, 14586-14591.

Huber, L., \& Gajdon, G. K. (2006). Technical intelligence in animals: The kea model. Animal Cognition, 9, 295-305.

Jensen, K., Hare, B., Call, J., \& Tomasello, M. (2006). What's in it for me? Self-regard precludes altruism and spite in chimpanzees. Proceedings of the Royal Society B: Biological Sciences, 273, 1013-1021.

Koyama, N., Caws, C., \& Aureli, F. (2006). Interchange of grooming and agonistic support in chimpanzees. International Journal of Primatology, 27, 1293-1309.

Lambert, M. L., Massen, J. J. M., Seed, A. M., Bugnyar, T., \& Slocombe, K. E. (2017). An 'unkindness' of ravens? Measuring prosocial preferences in Corvus corax. Animal Behaviour, 123, 383-393.

Marshall-Pescini, S., Dale, R., Quervel-Chaumette, M., \& Range, F. (2016). Critical issues in experimental studies of prosociality in non-human species. Animal Cognition, 19, 679-705.

Massen, J. J. M., Lambert, M., Schiestl, M., \& Bugnyar, T. (2015). Subadult ravens generally don't transfer valuable tokens to conspecifics when there is nothing to gain for themselves. Frontiers in Psychology, 6, 885. https://doi.org/10.3389/fpsyg.2015.00885

Massen, J., Luyten, I., Spruijt, B., \& Sterck, E. (2011). Benefiting friends or dominants: Prosocial choices mainly depend on rank position in long-tailed macaques (Macaca fascicularis). Primates, 52, 237-247.

Massen, J. J. M., Van den Berg, L. M., Spruijt, B. M., \& Sterck, E. H. M. (2010). Generous leaders and selfish underdogs: Pro-sociality in despotic macaques. PLoS One, 5, e9734.

Melis, A. P., Warneken, F., Jensen, K., Schneider, A., Call, J., \& Tomasello, M. (2011). Chimpanzees help conspecifics obtain food and non-food items. Proceedings: Biological Sciences, 278, 1405-1413.

Nissen, H., \& Crawford, M. (1936). A preliminary study of food-sharing behavior in young chimpanzees. Journal of Comparative Psychology, 22, 383-419.

Péron, F., John, M., Sapowicz, S., Bovet, D., \& Pepperberg, I. (2013). A study of sharing and reciprocity in grey parrots (Psittacus erithacus). Animal Cognition, 16, 197-210.

Quervel-Chaumette, M., Dale, R., Marshall-Pescini, S., \& Range, F. (2015). Familiarity affects other-regarding preferences in pet dogs. Scientific Reports, 5, 18102.

Schino, G. (2007). Grooming and agonistic support: A meta-analysis of primate reciprocal altruism. Behavioral Ecology, 18, 115-120.

Schwab, C., Swoboda, R., Kotrschal, K., \& Bugnyar, T. (2012). Recipients affect prosocial and altruistic choices in jackdaws. Corvus monedula. PLoS One, 7, e34922.

Schwing, R., Jocteur, E., Wein, A., Noë, R., \& Massen, J. J. M. (2016). Kea cooperate better with sharing affiliates. Animal Cognition, 19, 1093-1102.

Sebastián-Enesco, C., \& Warneken, F. (2015). The shadow of the future: 5 -Year-olds, but not 3-year-olds, adjust their sharing in anticipation of reciprocation. Journal of Experimental Child Psychology, 129, 40-54.

Silk, J. B., Brosnan, S. F., Vonk, J., Henrich, J., Povinelli, D. J., Richardson, A. S., ... Shapiro, S. J. (2005). Chimpanzees are indifferent to the welfare of unrelated group members. Nature, 437(7063), 1357-1359.

Skoyles, J. R. (2011). Chimpanzees make mean-spirited, not prosocial, choices. Proceedings of the National Academy of Sciences, 108(42), E835-E835.

Smith, J. D., Shields, W. E., \& Washburn, D. A. (2003). The comparative psychology of uncertainty monitoring and metacognition. Behavioral and Brain Sciences, 26, 317-339.

Stevens, J. (2010). Donor payoffs and other-regarding preferences in cotton-top tamarins (Saguinus oedipus). Animal Cognition, 13(4), 663670. https://doi.org/10.1007/s10071-010-0309-x 
Suchak, M., \& de Waal, F. B. M. (2012). Monkeys benefit from reciprocity without the cognitive burden. Proceedings of the National Academy of Sciences of the United States of America, 109, 15191-15196. https:// doi.org/10.1073/pnas.1213173109

Takimoto, A., Kuroshima, H., \& Fujita, K. (2010). Capuchin monkeys (Cebus apella) are sensitive to others' reward: An experimental analysis of food-choice for conspecifics. Animal Cognition, 13, 249-261.

Team, R. C. (2014). R: A language and environment for statistical computing. Vienna, Austria: R Foundation for Statistical Computing. http:// www.R-project.org/.

Tebbich, S., Taborsky, M., \& Winkler, H. (1996). Social manipulation causes cooperation in keas. Animal Behaviour, 52, 1-10.

Tennie, C., Jensen, K., \& Call, J. (2016). The nature of prosociality in chimpanzees. Nature Communications, 7. https://doi.org/10.1038/ncomm s13915

Vonk, J., Brosnan, S. F., Silk, J. B., Henrich, J., Richardson, A. S., Lambeth, S. P., ... Povinelli, D. J. (2008). Chimpanzees do not take advantage of very low cost opportunities to deliver food to unrelated group members. Animal Behaviour, 75, 1757-1770.

Warneken, F., Hare, B., Melis, A. P., Hanus, D., \& Tomasello, M. (2007). Spontaneous altruism by chimpanzees and young children. PLoS Biology, 5, e184.
Warneken, F., \& Tomasello, M. (2013). The emergence of contingent reciprocity in young children. Journal of Experimental Child Psychology, 116, 338-350.

Wascher, C. A. F., Feider, B., Bugnyar, T., \& Dufour, V.. (2019). Corvids fail to give and reciprocate in a token exchange task. Ethology.

Yamamoto, S., Humle, T., \& Tanaka, M. (2009). Chimpanzees help each other upon request. $P$ LoS One, 4, 1-7.

Yamamoto, S., Humle, T., \& Tanaka, M. (2012). Chimpanzees' flexible targeted helping based on an understanding of conspecifics' goals. Proceedings of the National Academy of Sciences, 109(9), 3588-3592. https://doi.org/10.1073/pnas.1108517109

How to cite this article: Heaney M, Bastos APM, Gray RD, Taylor AH. Are kea prosocial? Ethology. 2020;126:176-184. https://doi.org/10.1111/eth.12944 